\title{
Health Management Model: A Case Study for Success
}

\author{
Prosper M. Bernard \\ Department of Management, University of Québec in Montreal, QC, Canada
}

Received February 8, 2021; Revised March 12, 2021; Accepted April 9, 2021

\section{Cite This Paper in the following Citation Styles}

(a): [1] Prosper M. Bernard , "Health Management Model: A Case Study for Success," Universal Journal of Public Health, Vol. 9, No. 2, pp. 51 - 56, 2021. DOI: 10.13189/ujph.2021.090203.

(b): Prosper M. Bernard (2021). Health Management Model: A Case Study for Success. Universal Journal of Public Health, 9(2), 51 - 56. DOI: 10.13189/ujph.2021.090203.

Copyright $(2021$ by authors, all rights reserved. Authors agree that this article remains permanently open access under the terms of the Creative Commons Attribution License 4.0 International License

\begin{abstract}
Obesity is an important health issue in the current day and age. As it has been reported by many studies on the COVID-19 pandemic, individuals with excess weight and people with poor health are at a higher risk for COVID-19 severity. The purpose of this research is to give a model for losing weight by selecting a plant-based diet and by the progress measuring every day. The methodology is a case study with observations over three years. A case study is used to analyze the model and to teach in many disciplines in universities such as medicine, management, chiropractic, dentistry, psychology. Based on the findings of prior empirical studies, the subject made dietary and behavioral changes and was able to lose $45 \mathrm{~kg}$ (95 pounds) in approximately 18 months and maintain the proper weight for two years. The main conclusion is that by following this model including nutrition changes and regular measuring, people can reduce their weight to the normal level and keep it for a long period, hopefully, for the rest of life. This study shows how to change to plant-based food, avoid processed food, and measure the progress every day to adjust the behavior. Furthermore, the study shows that it is not a special diet but a long-term lifestyle. This study has limitations because a case study is not the same as a study with a randomized large sample. However, it contributes to the field of nutrition by showing that with a plant-based diet and a minor behavioral change, one can greatly improve his or her health by staying at a normal weight. This type of nutrition has a major social impact especially at this time of the COVID-19 pandemic because it affects particularly people in poor health conditions.
\end{abstract}

Keywords Obesity, Weight Loss, Behavior, Diet, Nutrition, Case Study

\section{Introduction}

Obesity is an important risk factor for many diseases, particularly for COVID-19, as shown by current studies $[1,22]$. Therefore, it is important to reduce weight in a natural manner. However, the global problem of obesity, particularly in Western countries, is on the rise since the 1980 [19]. Mercola [12] reports that American Obesity Association predicted the prevalence of obesity in half of the American population by 2025 (in only 4 years from now) and that this proportion can go up to $60 \%$ by 2030 . The author is not making any medical recommendation or any medical prescription.

\section{Objective}

The purpose of this article is to give the result of a case study of a healthy individual with an elevated BMI, who was able to lose $0.96 \mathrm{~kg}$ ( 2 pounds) per week, by making behavioral and dietary changes. The article is also describing the model in more details [2]. In this case study, the participant (the author) was able to reduce more than 45 $\mathrm{kg}$ (95 pounds) or $40 \%$ of the starting weight - in 18 months and maintain it for 2 years. This article also gives a model for success of being healthy while respecting the environment.

\section{Methodology}

This article is based on the case study method. A case study is a valid tool for research [23]. It is also a major tool 
for teaching in many disciplines such as medicine, chiropractic, dentistry, clinical psychology, business, and many other disciplines. A case study involves an in-depth investigation of a particular situation that can be generalized to many other situations. Therefore, it enlightens other people or organizations about the environment, to make decisions, take actions, analyze important variables, and evaluate its application for other cases. A case study is used to identify relevant variables or patterns that can be applied to a different environment. Case studies are used to study complex situations and to identify the relevant factors. Many schools, such as Harvard University, primarily use case studies as a teaching tool. The person observed in this case study is referred to as the subject. He is a male, in his 70s, and has been diagnosed with weight issues for the last 40 years. This case study confirms previous studies $[9,16]$. One must learn by doing - not only by listening to experts [17]. However, one needs medical support before doing any experiment. The scientific and medical foundations are explained ahead $[8,11,12]$. This case study involves two fundamental components. The key is implementing behavioral and dietary changes [7,12].

The subject has always reported being healthy, despite his excessive weight for many years. However, in January 2018, the weight was 268 pounds, which was 95 pounds over the target weight. Effective changes were needed to reach the Body-Mass Index (BMI) of 25 or below, with a weight of $78.5 \mathrm{~kg}$ (173 pounds [3]. The subject was the youngest in a large family of 13 . He was raised on a farm, which provided access to plenty of fresh produce. Although he was unaware of the term "organic," almost everything the family consumed was organic.

A limitation of some previous studies is that they use "death" as an indicator of poor health [8]. Quality of life is a more suitable measure of health. People in good health do not suffer toward the end of life. People who have a plant-based diet die at an incredibly old age and without suffering [8]. Most of the centenarians and super centenarians are quite healthy until the very end of their lives [13]. Many people blame the genes. It is incorrect to blame genes; it is rather the lifestyle and the food we eat [7].

\section{Discussion}

Why do people have weight problems? McDougall [11] explains that the main reason for this is the food that people eat. We can summarize it this way: "IT IS THE FOOD." It is believed by many that the causes of obesity could be linked to genetic, metabolic, and other factors. The answer is quite simple: It is the food that we eat [9]. Some individuals report eating unhealthy food, although they may not experience weight problems. However, they may have other health issues [11]. We are not all equal in life.
The same is true for everything. Some people have received a lot of money from their family, and other people have nothing. Some people have problems that others do not have. Since the subject had a weight problem that other people did not have, he decided to solve it like in any other domain.

The reason for the current problem is suggested to be the food that we eat. There is a simple solution to obesity $[11,12]$. The type of food is the reason for obesity. The solution is to eat the correct quality of food, rather than reducing its quantity. In this article the author refers to the research on plant-based diet [10].

\subsection{Medication to Lose Weight}

The subject had tried many things over the years with successes and failures. Successes were only short terms. One of the previous methods was a system of daily injections under medical supervision. The doctor told him that medications are effective for some people and not effective for others. It did work for a period, but after a while, the subject started to gain weight using the injections instead of reducing weight. Ultimately, he stopped all medications.

\subsection{Why Was the Subject Overweight?}

The subject opted for vegetarianism, 35 years ago. The goal at that time was not weight loss. His main reasons for adopting a vegetarian diet were related to the morality of killing animals and not preferring the taste of meat. He personally believes that eating meat is not very efficient from the perspective of food production. Moreover, raising cattle is not beneficial for the environment since it is an important contributor of greenhouse gases. Rearing cattle produces more greenhouse gases than driving cars [15]. In fact, rearing cattle is responsible for $16 \%$ of greenhouse gases, while transportation is responsible for $13.5 \%$ [11]. We are eating our planet to death. Thus, choosing a plant-based diet is a moral issue.

\subsection{The Trigger}

Everyone reaches a limit in their endeavors in the current state of things. In this case, it is when the subject needed to buy a suit and had to go to "special" store for "oversized" people. This was not a good experience.

\subsection{Simple Model}

Bernard [2] provides extensive scientific support for this model. The goal was to lose $11 \mathrm{~kg}$ ( $1 / 4$ pound) per day on average, which is approximately 2 pounds a week which is compatible to the norms $[9,13]$. This program consists of three components:

- Measuring every day. 
16 hours a day of fasting.

Plant-based food.

Measuring on the scale every day. The subject weighed himself every morning. The subject found that if he does not get on the scale every morning, the model does not work. He repeatedly travels to Asia (except in 2020 and 2021 because of COVID-19) and carries a portable scale. Weighting is emerging as the recommended practice for weight loss [18]. This is likely because it improves engagement in weight control behaviors. In business schools as well as in medical schools, professors teach that regular measuring is essential. One must measure on a regular basis, to repeat and control a process and make the adjustments quickly. It is necessary to measure it, to see if the process is on track and to be able to continue it. Furthermore, it is a major motivator.

Fasting 16-18 hours per day - The subject skipped supper so that he can fast for 16-18 hours per day, except for when traveling.

Plant-based alimentation - The subject has been vegetarian for more than 35 years. However, he liked cheese and other dairy products very much. For this process, he eliminated all dairy products except for a small amount of milk in coffee, when traveling.

The revised menu and schedule are as follows:

- Early morning: Coffee at 05:15.

- Breakfast: 06:30 or 07:30.

- Oatmeal and fruit.

- $\quad$ Some vitamin supplements.

- Lunch: 12:00 or 12:30.

- Baked potato and/or rice or pasta (better potatoes).

- Vegetable soup.

- Vegetables or legumes such as beans.

- $\quad$ Sometimes an apple or other fruit.

\section{- Dinner: None.}

He successfully anticipates the next meal for tomorrow instead of eating.

The subject followed these principles:

- Plant-based food.

- No animal products.

- No eggs. No dairy. Just a small amount of milk in coffee when traveling.

- Soy (or almond/coconut) milk instead of milk.

- No oil.

- No added sugar in food.

- Do not drink calorie-dense drinks.

- Look at calories per volume, instead of total calories.

- No food with sugar as important ingredient.

- No processed food as far as possible.

- Vegetables or legumes such as beans.

- Potatoes and soup for lunch as much as possible.

- Vitamins and mineral supplements.

- One or two prunes per day.

- Water with apple cider vinegar.

\subsection{Measuring Is Important}

Every morning, he measured his weight and entered it in EXCEL sheet to compare it with the objective. Recording the progress on an Excel sheet is an important motivator. He also weighed himself in the evening. Typically, there was a difference of 2-3 pounds between the previous evening and the next morning. Since reaching the goal weight 18 months ago, he now writes his weight every morning to see if he continues maintaining control. Now, measuring once per day in the morning is enough. However, it must be every day.

\subsection{Sleeping Is Important}

The subject found out that sound sleep is especially important for weight loss. This conclusion is consistent with other studies [21]. The subject often travels to China. He makes sure to sleep 8 hours in the plane, to keep the routine. This is also to eliminate the jet lag that would disturb the sleeping pattern and lead to excessive eating. In general, the subject went to bed before 9 p.m. and got up before 5 a.m. This is the most effective schedule compatible with fasting for 16 hours. This schedule is for the subject. It is not a recommendation.

\subsection{One Setback and Temptation}

However, the biggest temptation is not only eating more but skipping the scale. It is important to weigh oneself every morning. One must always measure himself or herself, on any system, to check whether he or she is on track. Johnson [5] shows that one must measure himself or herself every day to see if one is in control: "Smell the cheese to see if it is getting old".

\subsection{Following the Plan is Difficult}

There is the plan and there is the reality. One must always come back to the plan despite facing issues. The plan must be monitored; this is what we teach in management and in the medical field. One must look at quality rather than quantity. For people who have an issue with weight and food, there are two ways to look at food and calories. Try to eat fewer calories by portion control or eat the proper food. The case study recommends the latter.

\subsection{The Results}

On January 8, 2018, his weight was $121.56 \mathrm{~kg}$ (268 pounds) and his BMI was 38.1. In September 2019, he weighed 78.9 kilos (174 pounds), with a BMI less than 25. Figure 1 below gives the trend over time for the weight. Figure 2 gives the changes in the Body Mass Index (BMI) for the same period. It is important to notice that the model had no negative effect on the organ functions of the subject. For example, another positive effect was that the subject blood pressure went down significantly. 


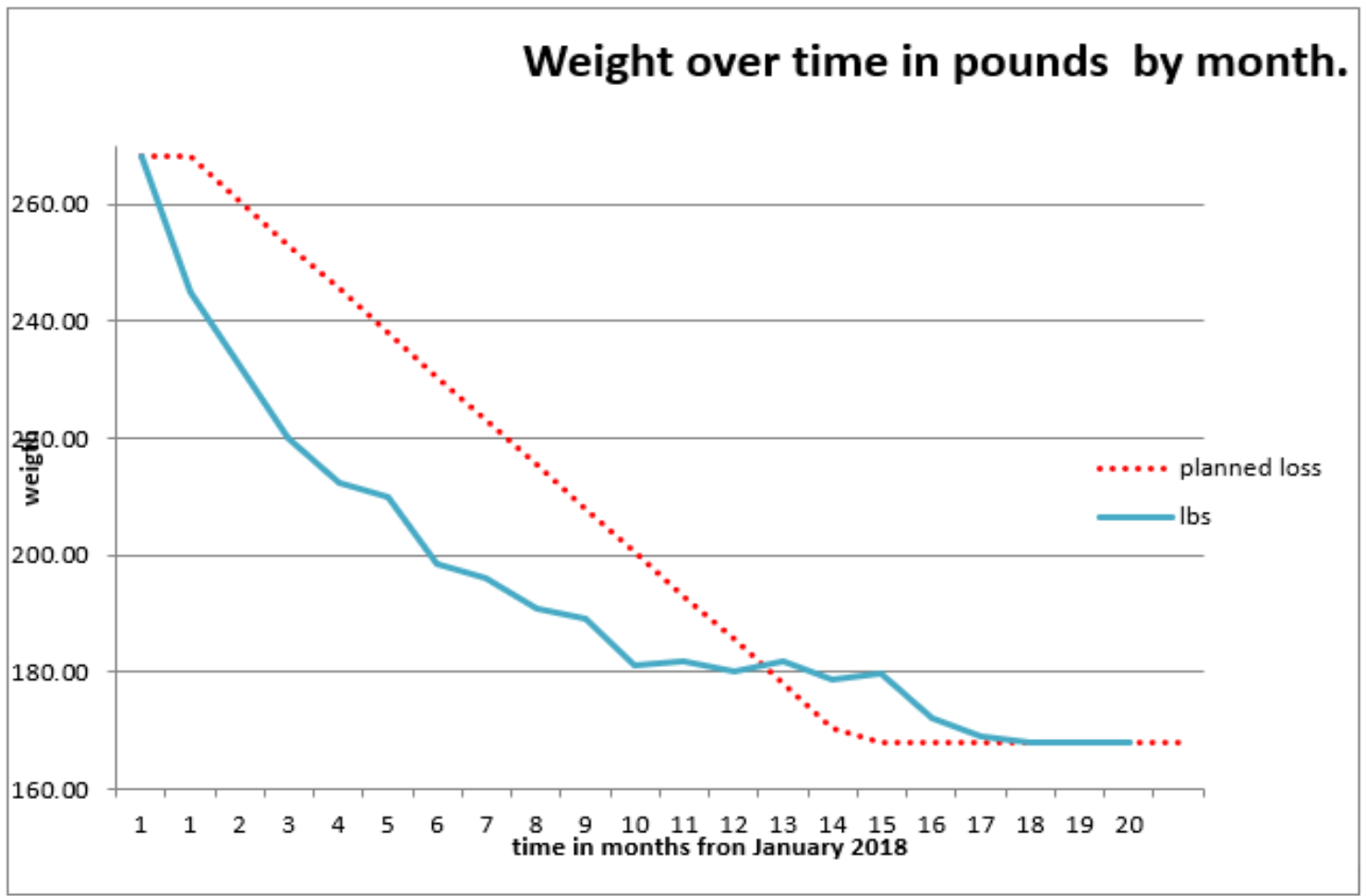

Figure 1. Weight (in pounds) over months

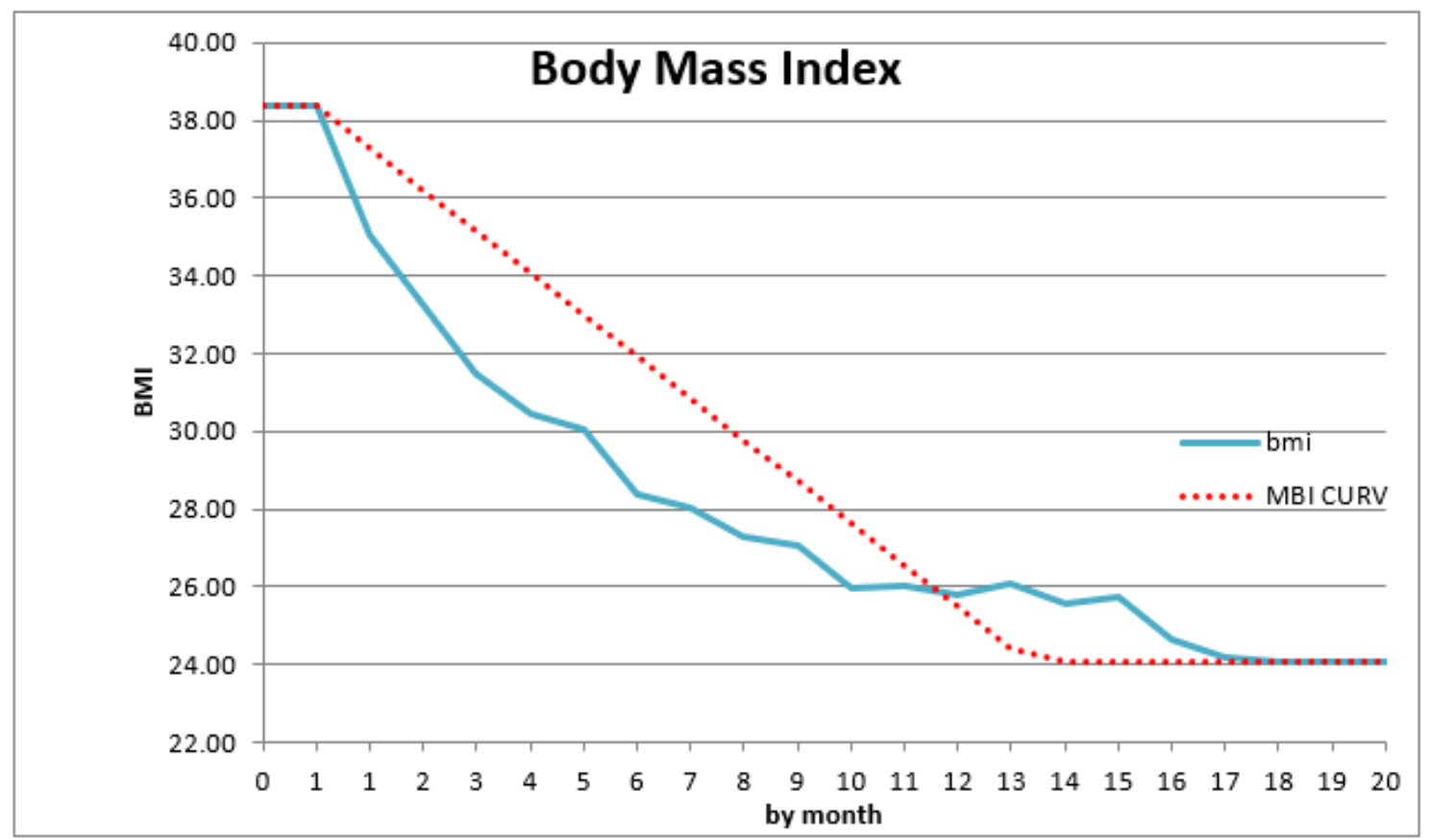

Figure 2. Body mass index tracked over months

\subsection{Scientific Basis in the Literature}

In this research, the recommendations given by multiple experts were tested with success $[9,13]$. Both medical researchers have extensively promoted natural food in their published works. They strongly suggest avoiding sugar. They also share much of the same vision about food and obesity: "IT IS THE FOOD". The subject has learned and applied models $[9,13]$. The case study goes one step further by combining plant-based diet, intermittent daily fasting, and daily measuring. One can subscribe to the above-described publications and daily messages $[11,14]$. There is always something that one can use. The main message is that one must eat the proper food instead of 
trying to eat less of "improper" food.

McDougall [11] has a remarkably interesting approach to promoting a plant-based diet. His view is that lecturing is not enough. He conducts a 10-day or 3-day working seminar in Santa Rosa, California, where he feeds participants 3 meals a day and involves them in the process. The subject has been to this on-site session in California. Unfortunately, because of the pandemic, these sessions were made offered in a virtual format in 2020 and 2021. This researcher believes that one must not only listen, but also be involved in the process. The subject has been to that seminar [10]. He tested with great success this model:

- A starch-based diet is needed to live healthy.

- Select food low in calorie concentration.

- No fats because the fat accumulates in the body.

- Eat foods high in complex carbohydrates because carbohydrates satisfy the hunger drive.

- Eat starches, vegetables, and fruits, and foods low in fat and high in carbohydrate.

- No meat, no dairy products, and no vegetable oil.

- Avid calorie dense, high fat, and low in carbohydrate

- Consume starches that are low in calories such as beans, peas, lentils, and potatoes.

- No sugar, no flour.

- Eat green and yellow vegetables.

- Eat more raw foods.

- No fruit juices.

- Eat rice cakes with buckwheat or corn. Rice pasta or whole wheat pasta.

- Soy products.

- No processed food.

- No alcohol.

Longo[18] explains that the main purpose of plant-based diet described above is not to live forever but to live up to an incredibly old age and dying without suffering. He promotes quality of life as a measure of success. He studied people who were over 100 years old. They were all healthy and mainly followed a plant-based diet. They died without suffering.

\subsection{Is Fasting Good for Health?}

Fasting has many health benefits including lowering blood sugar levels and promoting body fat loss [7]. Medical students receive only a few hours of lecturing and training on different aspects of nutrition including fasting [7].

\subsection{A Case Study on Potatoes to Confirm This Study}

There is a remarkably interesting case study to show that potatoes are a perfect food to lose weight [20]. In this study, the subject ate only potatoes for almost a year and was able to lose 110 pounds. Potatoes are the perfect item to reduce hunger and obtain all the needed nutrients [24,9].

\section{Conclusions}

The models given by multiple experts were tested with success $[9,13]$. The model is a plant-based diet with daily measuring. This case study showed that this model was significantly effective and easy to follow for the subject. However, one must do it when he or she is ready and has weight loss goals. One must monitor his or her progress every day. This case study also shows that the model can be sustained over time. It is not a quick diet. It is a lifestyle.

This model also had other benefits. It has allowed the subject to donate a lot of good clothing to a charity, maintain proper weight for two more years and most likely for life, look younger, avoid health complications, reduce the negative impact of COVID-19 in 2020-and 2021.

This study has limitations like most case studies. It is not a randomized sample in which many participants follow one model and others follow another. Such type of research study for single case could not be generalized unless we have a larger sample. However, it serves to confirm the theories expressed by important medical researchers $[9,12,8]$. The author did not study the effect of using regular food versus organic food. This would be useful to do in the future.

\section{Funding}

This project received no specific grant from any funding public agency, nor any private organization.

\section{Conflict of Interest}

The author declares that he has no conflict of interest.

\section{REFERENCES}

[1] Alberca RW, Oliveira LDM, Branco ACCC, Pereira NZ and Sato MN, "Obesity as a Risk Factor for COVID-19: An Overview," Critical Reviews in Food Science and Nutrition, vol. 1, no. 15, online, 2020. DOI:10.1080/10408398.2020.1 775546 .

[2] Bernard P, Plaisent M., Health Management models, A case study for success, University Consortium, Sarasota, Florida, USA, 2019.

[3] Dansinger, Michael, "Weight Loss and Body Mass Index (BMI)," WebMD Medical reference, https://www.webmd.c om/ (retrieved: 2021.01 30).

[4] Fung J, Moore J, The Complete Guide to Fasting, Victory Belt Publishing, Canada, 2019.

[5] Johnson, Spencer, Who Moved My Cheese?, Penguin Putman Inc. 2002.

[6] Kuk JL, Rotondi M, Sui X, Blair SN and Ardern CI., 
"Individuals with Obesity But No Other Metabolic Risk Factors Are Not At Significantly Elevated All - Cause Mortality Risk In Men And Women". Clinical Obesity, Wiley Online Library vol. 8, no. 5, pp. 305-312, 2018. DOI: 10.1111/cob.12263,

[7] Larocque M., L'obésité n'est pas une maladie, Éditions Un Monde Différent, Brossard, Qc, Canada. 2020.

[8] Longo V., The Longevity Diet, Avery, New York, 2018.

[9] McDougall J, McDougall M., The Starch Diet., Rodale, Pennsylvania, 2012.

[10] McDougall J, McDougall M., McDougall Program for Maximum Weight Loss, Plume, New York, 2013. https://www.drmcdougall.com/ (Accessed 2021.01.20)

[11] McDougall J, McDougall M., The Healthiest Diet on The Planet, Harper One, California, 2016.

[12] Mercola J., Fat for Fuel, Hay House, Carlsbad, California, USA, 2017.

[13] Mercola J., Simple Strategies to Live Longer, Online, 2019. https://www.mercola.com/ (Accessed 2021.02.04).

[14] Mercola J., 60\% of Americans will be Obese by 2030, Publications of Dr. Joseph Mercola, 2021, available on https://www.mercola.com/ (Accessed 2021.02.03].

[15] Fiala, Nathan, "The Greenhouse Hamburger," Scientific
American, vol. 300, no. 2, 2009, pp. 72-75.

[16] Rosati K, Gurkin K., The Rice Diet Renewal, Wiley, New Jersey, 2010.

[17] Roberts, Jay, Beyond Learning by Doing. Routledge, New York, 2012.

[18] Steinberg DM, Bennett GG, Askew S and Tate DF., "Weighing Everyday Matters: Daily Weighing Improves Weight Loss and Adoption of Weight Control Behaviors," Journal of the Academy of Nutrition and Dietetics, vol. 11, no, 4, pp. 511-518, 2015.

[19] Taubes, Gary, "As Obesity Rates Rise, Experts Struggle to Explain Why," Science, Vol. 280, Issue 5368, pp. 1367-1368, 1998. DOI: 10.1126/science.280.5368.1367.

[20] Taylor A, The Spud Fit Challenge, Spud Fit Taylor, Australia, 2016.

[21] Valrie CR, Bond K, Lutes LD, Carraway M and Collier DN, "Relationship of Sleep Quality, Baseline Weight Status, and Weight-Loss Responsiveness In Obese Adolescents," Sleep Medicine, Vol. 16, no. 3, pp.432-434, 2015.

[22] Yang J, Hu J and Zhu C., "Obesity Aggravates COVID - 19: A Systematic Review and Meta - Analysis," Journal of Medical Virology, Vol. 93, No. 1, pp. 257-261, 2021.

[23] Yin RK, Case Study Research, Sage Publications. Thousand Oaks, California, 2003. 